\title{
Cooperative Relaying with CPFSK and Distributed Space-Time Trellis Codes
}

\author{
Rachel L. Maw, Philippa A. Martin, Senior Member, IEEE, and Desmond P. Taylor, Life Fellow, IEEE
}

\begin{abstract}
Cooperative relaying allows single antenna users to achieve diversity and coding gains by utilizing nearby users' transmitting capabilities. We consider a relay system employing constant envelope continuous phase frequency shift keying. Distributed space-time trellis codes are implemented with a novel multiple relay protocol.
\end{abstract}

Index Terms-Cooperative relays, space-time trellis codes, continuous phase modulation.

\section{INTRODUCTION}

$\mathbf{C}$ OOPERATIVE relaying can realize the advantages of space-time coding without requiring a user to have multiple transmit antennas. Continuous phase modulation (CPM) is a non-linear modulation [1]. It has the advantage over linear modulations of a constant envelope, which allows the use of low cost and efficient non-linear power amplifiers. It has thus far not been considered in a cooperative relaying context, but has been shown to be a good alternative to linear modulation in space-time coded systems [2], [3], [4]. Continuous phase frequency shift keying (CPFSK) is a special case of CPM [1]. Here we consider CPFSK modulated cooperative relaying schemes.

There are many possible cooperative relaying configurations [5], [6], [7], [8]. We consider half duplex schemes, where a relay can receive and transmit, but not at the same time. They may be classified as either amplify and forward (AF) or decode and forward (DF) schemes. In AF schemes, the relays simply amplify received signals by a gain factor $\beta$ and retransmit them in subsequent time slots. In contrast, DF strategies require the relay terminals to have knowledge of the source's modulation format and coding to regenerate signals. AF schemes are, therefore, more suited to ad-hoc implementation. We investigate single-user AF relay schemes.

Three protocols for 1-relay systems transmitting over two time slots are defined in [5] and are described in Table I. Here a system consists of 3 nodes, a source $(\mathcal{S})$, relay $(\mathcal{R})$ and destination $(\mathcal{D})$. In [9], the protocol I AF cooperative scheme is shown to be optimal in terms of the diversity/multiplex tradeoff (DMT). In order to decode, the destination must know $\beta$ and the channel gains, $h_{\mathcal{S R}}, h_{\mathcal{S D}}$ and $h_{\mathcal{R D}}$, for the links $\mathcal{S} \rightarrow \mathcal{R}, \mathcal{S} \rightarrow \mathcal{D}$ and $\mathcal{R} \rightarrow \mathcal{D}$, respectively. The DMT can be used as a design tool. It shows which scheme will be superior given sufficient SNR, but not what SNR is sufficient [9].

Protocol I AF is extended to allow multiple relays in [9]. Each relay receives a source transmission in turn (every second

Manuscript received February 1, 2008. The associate editor coordinating the review of this letter and approving it for publication was G. K. Karagiannidis.

The authors are with the Department of Electrical and Computer Engineering, University of Canterbury, Private Bag 4800, Christchurch, New Zealand (e-mail: rlm57@student.canterbury.ac.nz, taylor@elec.canterbury.ac.nz).

Digital Object Identifier 10.1109/LCOMM.2008.080163.
TABLE I

Established Single Relay Cooperative Protocols [5].

\begin{tabular}{|c|c|c|c|c|}
\hline & \multicolumn{2}{|c|}{ Nodes Transmitting } & \multicolumn{2}{c|}{ Nodes Receiving } \\
\hline Time Slot & $\mathbf{1}$ & $\mathbf{2}$ & $\mathbf{1}$ & $\mathbf{2}$ \\
\hline Protocol I & $\mathcal{S}$ & $\mathcal{S}, \mathcal{R}$ & $\mathcal{R}, \mathcal{D}$ & $\mathcal{D}$ \\
\hline Protocol II & $\mathcal{S}$ & $\mathcal{R}$ & $\mathcal{R}, \mathcal{D}$ & $\mathcal{D}$ \\
\hline Protocol III & $\mathcal{S}$ & $\mathcal{S}, \mathcal{R}$ & $\mathcal{R}$ & $\mathcal{D}$ \\
\hline
\end{tabular}

time slot), then amplifies and forwards it in the next time slot. A system with $N$ relays requires $2 N$ time slots for each cooperation frame. Because only $\frac{1}{2}$ the information is repeated by the relays, the link cannot support multiplexing gains greater than $\frac{1}{2}$. In [10], [11] slotted AF (SAF) schemes are developed that have improved DMTs for $N>1$. The focus is on the naive SAF protocol, where each relay listens in turn for 1 slot to receive the source and previous relay's transmissions and then amplifies and forwards them in the next slot. For $N$ relays there are $N+1$ slots per cooperation frame. The last relays are receiving highly corrupted signals from the previous relays, as is the destination. This protocol can be optimal in terms of the DMT with an infinite number of slots if the relays are physically isolated or if ordering or dumping of relays based on their channel quality is used [10]. Improving capacity by allowing multiple users to use the same relay slot is investigated in [12], but results in inter-user interference during the communal slot.

In Section II we propose novel AF protocols for multiple relays. In Section III and IV we present and simulate a relaying scheme employing CPFSK and distributed space-time trellis codes (DSTTCs). Conclusions are drawn in Section V.

\section{Multiple Relay Protocols}

We propose a novel extension to the three protocols of Table I for multiple relays, as illustrated by Table II. Here each protocol employs $N$ relays and requires $N+1$ intervals for one cooperation frame. An interval can range from a single symbol period up to a complete data frame. Relays 1 to $N$ are labelled $\mathcal{R}_{1}, \ldots, \mathcal{R}_{N}$. The source transmits for the first $N$ or $N+1$ intervals depending on the protocol. Each relay listens for an interval in turn over the first $N$ intervals. All relays forward their amplified received signal to the destination in the $N+1$-th interval. For EXT-I, $\frac{N}{N+1}$ of the source information is forwarded by the relays, allowing multiplexing gains up to $\frac{N}{N+1}$, the same as the naive SAF protocol. Beneficially, the proposed protocols do not suffer from the extensive error propagation inherent to the naive SAF protocol, as no relay listens to other relays' forwarded messages. The DMT is optimized using EXT-1. In the case of 1 relay the protocols of 
TABLE II

Proposed Cooperative Relaying Protocols for Systems with $N$ Relays

\begin{tabular}{|c|c|c|c|c|c|c|c|c|c|c|}
\hline & \multicolumn{5}{|c|}{ Nodes Transmitting } & \multicolumn{5}{|c|}{ Nodes Receiving } \\
\hline Interval & 1 & 2 & $\ldots$ & $\mathbf{N}$ & $\mathrm{N}+1$ & 1 & 2 & $\ldots$ & $\mathbf{N}$ & $\mathbf{N}+1$ \\
\hline Protocol EXT-I & $\mathcal{S}$ & $\mathcal{S}$ & $\ldots$ & $\mathcal{S}$ & $\mathcal{S}, \mathcal{R}_{1}, \mathcal{R}_{2}, \ldots, \mathcal{R}_{N}$ & $\overline{\mathcal{R}_{1}, \mathcal{D}}$ & $\overline{\mathcal{R}_{2}, \mathcal{D}}$ & $\ldots$ & $\mathcal{R}_{N}, \mathcal{D}$ & $\mathcal{D}$ \\
\hline Protocol EXT-II & $\mathcal{S}$ & $\mathcal{S}$ & $\ldots$ & $\mathcal{S}$ & $\mathcal{R}_{1}, \mathcal{R}_{2}, \ldots, \mathcal{R}_{N}$ & $\mathcal{R}_{1}, \mathcal{D}$ & $\mathcal{R}_{2}, \mathcal{D}$ & $\ldots$ & $\mathcal{R}_{N}, \mathcal{D}$ & $\mathcal{D}$ \\
\hline Protocol EXT-III & $\mathcal{S}$ & $\mathcal{S}$ & $\ldots$ & $\mathcal{S}$ & $\mathcal{S}, \mathcal{R}_{1}, \mathcal{R}_{2}, \ldots, \mathcal{R}_{N}$ & $\overline{\mathcal{R}_{1}}$ & $\overline{\mathcal{R}_{2}}$ & . . & $\mathcal{R}_{N}$ & $\mathcal{D}$ \\
\hline
\end{tabular}

Table II reduce to those of Table I. Note, the EXT-1 protocol has been proposed for multi-user differential distributed spacetime modulation in [13]. Using the approach of [13] all 3 protocols in Table II can be extended to a multi-user system.

\section{System Model}

We propose DSTTCs employing EXT-I and EXT-III based on the space-time coded $M$-CPFSK system of [14], with modulation index $h=\frac{1}{M}$. EXT-II is not considered because it does not utilize a source transmission in the final interval, which limits the rate of the DSTTC. In [14], CPFSK is modelled by the Rimoldi decomposition [15], consisting of a continuous phase encoder (CPE) followed by a memoryless modulator. $L_{t}$ feedback free CPEs are combined into a single convolutional encoder which has the $L_{t} \times 2 L_{t}$ generator matrix [14]

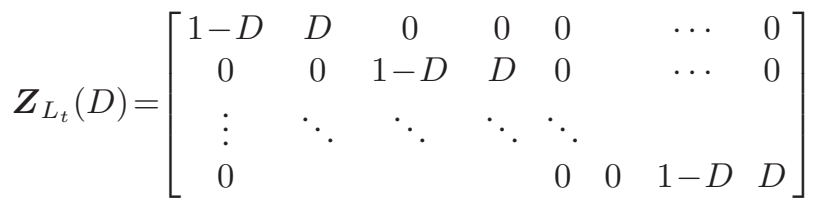

defined over the ring of integers modulo- $M\left(\mathbb{Z}_{M}\right)$, where $L_{t}$ is the number of transmit antennas. By considering rate- $\frac{L_{t}-1}{L_{t}}$ space-time codes (STCs) defined over $\mathbb{Z}_{M}$, the generators of the modulation, $\boldsymbol{Z}_{L_{t}}(D)$, and the STC, $\boldsymbol{G}(D)$, may be combined to form a trellis encoder with generator matrix $\boldsymbol{J}(D)=\boldsymbol{G}(D) \boldsymbol{Z}_{L_{t}}(D)$ on $\mathbb{Z}_{M}$.

Our distributed scheme employs $N$ relays and instead of transmitting from $L_{t}$ co-located antennas as for traditional space-time coding (TSTC), the source transmits the coded data over $L_{t}=N+1$ intervals. Here an interval is the number of symbol periods, $n_{f}$, required to transmit a data frame in a TSTC scheme. We consider a rate- $\frac{N}{N+1}$ STC giving a system throughput of $\frac{N \log _{2} M}{N+1}$ bits per channel use (BPCU) or symbol period. Note the TSTC has throughput $N \log _{2}(M)$ BPCU. The source has 1 transmit antenna and the relays each have 1 transceiver antenna. Thus, only 1 memoryless modulator is required at $\mathcal{S}$. Its input is formed by time-multiplexing the output of $\boldsymbol{J}(D)$ so that a frame of modulated data transmitted from the $i$-th antenna in the TSTC scheme is transmitted in the $i$-th interval for $1 \leq i \leq N+1$. There are $L_{r}$ receive antennas at $\mathcal{D}$. The maximum achievable diversity is upper bounded by $r L_{r}$, where $r$ is the transmit diversity of the STC.

The signal transmitted during the $n$-th symbol period of the $i$-th interval is given by

$$
s_{i}\left(t, \boldsymbol{X}_{i, n}\right)=\sqrt{\frac{2 E}{L_{t} T}} \cos \left(2 \pi f_{1} t+\tilde{\psi}\left(t, \boldsymbol{X}_{i, n}\right)\right),
$$

where the information carrying phase is defined as [15]

$$
\tilde{\psi}\left(t, \boldsymbol{X}_{i, n}\right)=\left[2 \pi h\left(X_{i, n}^{2}+X_{i, n}^{1} \frac{t-n T}{T}\right)\right] \bmod 2 \pi,
$$

$n T \leq t \leq(n+1) T$ in (2) and (3), $f_{1}$ is the asymmetric carrier frequency [15], $E$ is the energy per symbol period, $T$ is the symbol period and $\boldsymbol{X}_{i, n}=\left[X_{i, n}^{1} X_{i, n}^{2}\right]$ is the output from the $i$-th CPE [14]. We normalize the signal transmitted from the $i$-th relay using the amplification factor $\beta_{i}=\sqrt{\frac{\frac{E}{T}}{\frac{E}{T}\left|h_{S R_{i}}\right|^{2}+\sigma_{n}{ }^{2}}}$ for $1 \leq i \leq N$, where $\sigma_{n}{ }^{2}$ is the noise power and $h_{\mathcal{S} \mathcal{R}_{i}}$ is the fade coefficient of the $\mathcal{S} \rightarrow \mathcal{R}_{i}$ link. The average transmit power of each relay in the interval it transmits is then less than or equal to $\frac{E}{T}$, the transmit power of the source.

Perfect channel state information, $h_{\mathcal{S R}_{i}}, h_{\mathcal{S D}}, h_{\mathcal{R}_{i} \mathcal{D}}$, is assumed at $\mathcal{D}$, as is knowledge of the amplification factors $\beta_{i}$ for $1 \leq i \leq N$. Because the coded data is stored at the relays and transmitted simultaneously in the last interval, the trellis of $\boldsymbol{J}(D)$ is used for Viterbi decoding at $\mathcal{D}$. If EXT-I is used, the decoding metric at $\mathcal{D}$ is augmented to use the information received during the first $N$ intervals, and the received signal at the $j$-th antenna at $\mathcal{D}$ is

$$
\begin{gathered}
y_{j}(t, \boldsymbol{X})=\sum_{i=1}^{N}\left[\beta_{i} h_{\mathcal{R}_{i} \mathcal{D}}\left[h_{\mathcal{S R}_{i}} s_{i}\left(t, \boldsymbol{X}_{i}\right)+n_{\mathcal{R}_{i}}(t)\right]\right] \\
+h_{\mathcal{S D}} \sum_{i=1}^{N+1} s_{i}\left(t, \boldsymbol{X}_{i}\right)+n_{j}(t), j=1, \ldots, L_{r}
\end{gathered}
$$

where $n_{\mathcal{R}_{i}}(t)$ and $n_{j}(t)$ are the AWGN processes for the $i$-th relay and $j$-th antenna at $\mathcal{D}$, respectively. The metric is then

$$
\begin{aligned}
& M(y(t, \boldsymbol{X}) \mid \hat{\boldsymbol{X}})=-\sum_{j=1}^{L_{r}} \int_{0}^{n_{f}(N+1) T} \mid y_{j}(t, \boldsymbol{X})- \\
& \sum_{i=1}^{N}\left[\beta_{i} h_{\mathcal{R}_{i} \mathcal{D}} h_{\mathcal{S R}_{i}} s_{i}\left(t, \hat{\boldsymbol{X}}_{i}\right)\right]-\left.h_{\mathcal{S D}} \sum_{i=1}^{N+1} s_{i}\left(t, \hat{\boldsymbol{X}}_{i}\right)\right|^{2} d t .
\end{aligned}
$$

\section{NumERICAL RESUlTS}

In the following simulations we use 4-CPFSK and the data frames consist of 130 4-ary symbols. Quasi-static Rayleigh flat fading is assumed, so that the fading coefficients are constant over the cooperation frame of $N+1$ intervals required to send a data frame. They are modelled as complex Gaussian random variables with mean 0 and variance $\frac{1}{2}$ per dimension. We let the signal to noise ratio (SNR) of the links $\mathcal{R}_{i} \rightarrow \mathcal{D}$ for $1 \leq i \leq N$ and $\mathcal{S} \rightarrow \mathcal{D}$ be equal and denoted $\operatorname{SNR}_{\mathcal{D}}=$ $10 \log _{10}\left(\frac{E_{b}}{N_{0}}\right) \mathrm{dB}$, where $E_{b}$ is the bit energy and $N_{0}$ is the one-sided noise power spectral density. We assume the SNR of the links $\mathcal{S} \rightarrow \mathcal{R}_{i}$ for $1 \leq i \leq N$ to be $\mathrm{SNR}_{\mathcal{R}}=\mathrm{SNR}_{\mathcal{D}}$ $+10 \mathrm{~dB}$. This would be approximately true if the source and relays were near each other and the destination was located far away ${ }^{1}$. It was shown in [5] that TSTC design rules may

\footnotetext{
${ }^{1} \mathrm{SNR}_{\mathcal{R}}>\mathrm{SNR}_{\mathcal{D}}$ is required for relaying to offer significant benefits [5]
} 


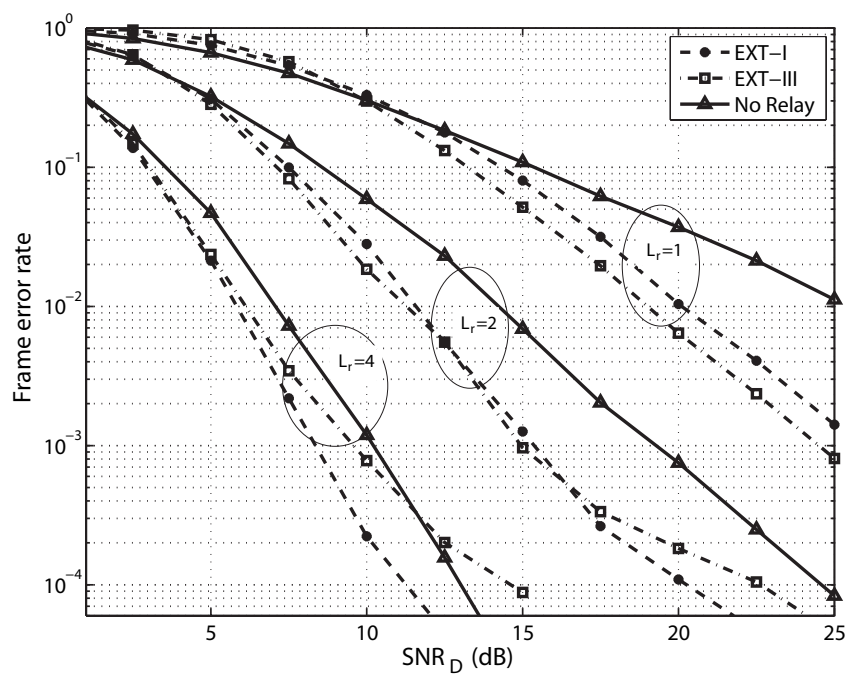

Fig. 1. FER of DSTTC with 4-CPFSK and 1 relay (1 BPCU).

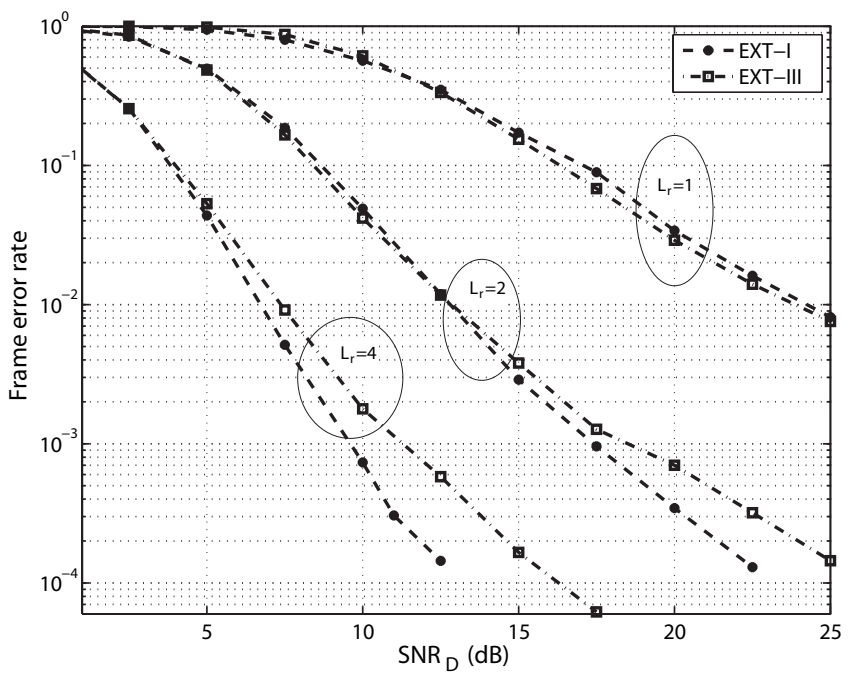

Fig. 2. FER of DSTTC with 4-CPFSK and 2 relays ( $\frac{4}{3}$ BPCU).

be used to design DSTTCs (with appropriate power control). In the examples, we use the optimized codes of ${ }^{2}$ [4].

Fig. 1 shows the frame error rate (FER) performance of 1-relay schemes using EXT-I and EXT-III, where $\boldsymbol{G}(D)=$ $\left[\begin{array}{ll}1 & \frac{2+D}{1+2 D}\end{array}\right], \boldsymbol{J}(D)=\left[\begin{array}{llll}1-D & D & \frac{2-D-D^{2}}{1+2 D} & \frac{2 D+D^{2}}{1+2 D}\end{array}\right]$ and $r=2$. The performance with no relay is also shown. Each scheme has a throughput of $1 \mathrm{BPCU}$, and is simulated with $L_{r}=1,2$ and 4 antennas at $\mathcal{D}$. For $L_{r}=1$, EXT-III has the best performance and both cooperative schemes provide greater diversity than using direct transmission. For $L_{r}=2$ and 4, EXT-I outperforms EXT-III at low FER and both start to exhibit reduced diversity due to the amplification of $n_{\mathcal{R}_{i}}$ over the $\mathcal{R}_{i} \rightarrow \mathcal{D}$ link. Increasing $\mathrm{SNR}_{\mathcal{R}}$ should delay this reduction. At very low FER with $L_{r}=4$ there is no improvement using EXT-III instead of direct transmission.

Fig. 2 shows the performance of cooperative relay schemes

\footnotetext{
${ }^{2}$ Despite not having full rank, the STCs in [4] were shown to provide excellent performance. For the same throughput, they outperform full rank delay diversity using CPFSK.
}

with 2 relays, where $\boldsymbol{G}(D)=\left[\begin{array}{ccc}1 & 0 & 2 D \\ 0 & 1 & 1+2 D\end{array}\right], \boldsymbol{J}(D)=$ $\left[\begin{array}{cccccc}1-D & D & 0 & 0 & 2 D-D^{2} & 2 D^{2} \\ 0 & 0 & 1-D & D & 1+D-2 D^{2} & D+2 D^{2}\end{array}\right]$ and $r=1$. The system throughput is $\frac{4}{3}$ BPCU. The relative performance of the two protocols is similar to that of the 1relay systems. The performance of the system with no relay is not shown as it is unusably bad.

\section{CONClusion}

We have presented a distributed space-time trellis coded system employing CPFSK and a novel multi-relay protocol. Performance gains are achieved using AF cooperative relaying. As expected EXT-I provides the best performance for $L_{r}=2$ and 4. Surprisingly EXT-III is superior for $L_{r}=1$ in the simulated range of $\mathrm{SNR}_{\mathcal{D}}$.

\section{REFERENCES}

[1] J. B. Anderson, T. Aulin, and C.-E. W. Sundberg, Digital Phase Modulation. New York: Plenum, 1986.

[2] M. P. Fitz and X. Zhang, "A case for continuous phase modulation in space-time modems," in Proc. Inform. Theory Workshop, Oct. 2002, pp. 61-64.

[3] J. K. Cavers, "Space-time coding using MSK," IEEE Trans. Wireless Commun., vol. 4, no. 1, pp. 185-191, Jan. 2005.

[4] R. L. Maw, "Space-time coded systems with continuous phase modulation," Ph.D. dissertation, Univ. of Canterbury, New Zealand, 2007. [Online]. Available: http://www.library.canterbury.ac.nz

[5] R. U. Nabar, H. Bolcskei, and F. W. Kneubuhler, "Fading relay channels: performance limits and space-time signal design," IEEE J. Select. Areas Commun., vol. 22, no. 6, pp. 1099-1109, Aug. 2004.

[6] J. N. Laneman and G. W. Wornell, "Distributed space-time-coded protocols for exploiting cooperative diversity in wireless networks," IEEE Trans. Inform. Theory, vol. 49, no. 10, pp. 2415-2425, Oct. 2003.

[7] Y. Jing and B. Hassibi, "Distributed space-time coding in wireless relay networks," IEEE Trans. Wireless Commun., vol. 5, no. 12, pp. 35243536, Dec. 2006.

[8] A. Nosratinia, T. E. Hunter, and A. Hedayat, "Cooperative communication in wireless networks," IEEE Commun. Mag., pp. 74-80, Oct. 2004.

[9] K. Azarian, H. El Gamal, and P. Schniter, "On the achievable diversitymulitplexing tradeoff in half-duplex cooperative channels," IEEE Trans. Inform. Theory, vol. 12, no. 51, pp. 4152-4172, Dec. 2005.

[10] S. Yang and J.-C. Belfiore, "Towards optimal amplify-and-forward cooperative diversity scheme," submitted to IEEE Trans. Inform. Theory.

[11] _ "On slotted amplify-and-forward cooperative diversity schemes," in Proc. ISIT, Seattle, USA, July 2006, pp. 2446-2450.

[12] O. Muñoz, A. Agustín, and J. Vidal, "Cellular-capacity gains of cooperative mimo transmission in the downlink," in Proc. Int. Zurich Seminar on Commun., Feb. 2004, pp. 22-26.

[13] G. Wang, Y. Zhang, and M. Amin, "Differential distributed space-time modulation for cooperative networks," IEEE Trans. Wireless Commun., vol. 5, no. 11, pp. 3097-3108, Nov. 2006.

[14] R. L. Maw and D. P. Taylor, "Space-time coded systems with continuous phase frequency shift keying," in Proc. GLOBECOM, vol. 3, Nov. 2005, pp. $1581-1586$.

[15] B. E. Rimoldi, "A decomposition approach to CPM," IEEE Trans. Inform. Theory, vol. 34, no. 2, pp. 260-270, Mar. 1988. 